\title{
Human Reliability Analysis (HRA) for surgery: A modified HEART application to robotic surgery
}

\author{
Paolo Trucco $^{1}$, Rossella Onofrio ${ }^{1}$, Antonio Galfano ${ }^{2}$ \\ ${ }^{1}$ Politecnico di Milano, Milan, Italy \\ \{Paolo.Trucco, Rossella.Onofrio\}@polimi.it \\ ${ }^{2}$ Ospedale Niguarda Ca' Granda, Milan, Italy \\ Antonio.Galfano@OspedaleNiguarda.it
}

\begin{abstract}
HRA studies in healthcare highlight that PSF (or Influencing Factors - IFs) taxonomies in HRA techniques have been developed and validated in industrial contexts, and as such are not fully applicable to healthcare contexts. In this paper, a modified version of Human Error Assessment and Reduction Technique (HEART), has been developed and tested through an application to the robotic surgical Radical Prostatectomy procedure. Personal and organizational factors were modeled and assessed through an IFs taxonomy validated in the surgical domain, and then systematically translated into the corresponding Error Producing Conditions (EPCs), typical of the HEART method. The results confirmed the importance of adapting HRA methods to the healthcare sector, and added detailed information on what are the most relevant factors that should be captured by an HRA method when applied to surgery. Additionally, the analysis revealed that team related factors have the highest influence on surgeons' performance (i.e. increase of Human Unreliability Rate) in the context of different surgical tasks.
\end{abstract}

Keywords: Human Reliability Analysis · HRA · surgery · influencing factors ·

\section{Introduction}

The benefits of transferring and applying to healthcare the most important proactive risk analysis methods traditionally implemented in industry are fully recognized in patient safety literature [1], [2], [3], [4]. Literature findings highlight the lack of knowledge about the reach spectrum of HRA techniques and methodologies that are applicable to the healthcare sector [1], [2], [3], [4]. On the other side, an emerging and increasing interest in HRA applications has been pointed out, especially in surgery [5], [6], [7], [8], [9]. Therefore, there seems to be a growing need of fostering theoretical knowledge and practical expertise on HRA application in healthcare, to achieve higher safety performance under increasing environmental complexity and technological innovation, such as in the case of mini-invasive surgery. One of the critical aspects come up from extant HRA applications in healthcare concerns the limited adoption or misuse of Performance Shaping Factors (PSF), i.e. aspects of behavior and context that impact human performance [10]. On the contrary, in HRA theory and applications in industry, contextual and organizational factors play a 
relevant role, and the attempt to incorporate them into new and more advanced HRA techniques represents a hot research line in this discipline since a long time.

A closer look to the few papers describing HRA applications in healthcare where human and organizational factors are included reveals that all the authors dedicated particular attention to the choice of the most appropriate influencing factors. This modeling effort generally culminated in the decision to take into account only some of the factors covered by the selected HRA technique. Nevertheless, almost all the authors highlight that PSF (or Influencing Factors - IFs) taxonomies in HRA techniques have been developed and validated in industrial contexts, and as such are not fully applicable to the healthcare contexts [5].

In order to foster the diffusion of HRA in healthcare it is not enough applying and adapting existing HRA techniques to the healthcare context. A proper translation of these techniques to healthcare environment has to be undertaken [11], [12]. To this end, particular attention should be addressed to the role of those human and organizational factors that could shape the performance of the operator (e.g. surgeon or anesthetists) under different contexts.

In this paper, a modified version of Human Error Assessment and Reduction Technique [13], has been developed and tested through an application to the robotic surgical Radical Prostatectomy procedure, that is the most widespread robotic surgical procedure.

\section{Method}

In line with much part of the worldwide technological evolution in surgery, Italy represents one of the leading countries in Europe for the implementation of robotic surgery, as one of the most sophisticated new frontier of Minimally Invasive Surgery. Robotic Surgery has established itself as the best technique for the surgical treatment of prostate cancer. Today, in the US, over $80 \%$ of prostatectomies are performed with the aid of the DaVinci Surgical System. Although robotic surgery, or robot-assisted surgery, allows doctors to perform many types of complex procedures with more precision, flexibility and control [14], the advanced technology may introduce new error pathways due to the high degree of human-machine interaction, in fact the assessment of safety, feasibility and clinical efficacy is still at an early stage of study and analysis. This is the reason why we chose to apply HEART methodology on the most widespread robotic surgical procedure, i.e. radical prostatectomy. In particular, the study focuses on the analysis of a recent robotic prostatectomy technique performed with DaVinci robot, which allows to obtain excellent results both in oncological and functional terms [15], [16].

\subsection{Modified HEART for integration with a new taxonomy of IFs in surgery}

Starting from a literature review on the applications of HRA techniques in healthcare, and HEART in particular, the need for some methodological modifications was identified to make the traditional HEART more suitable for applications in surgery. 
Firstly, to capture the surgical environment peculiarities, personal and organizational factors were modeled and assessed through a specific IFs taxonomy, designed and validated ad hoc for the surgical context [17] and then systematically translated into the weights of corresponding Error Producing Conditions (EPC), typical of the traditional HEART method [13]. The proposed taxonomy is composed by 20 Influencing Factors (IFs) and it is well-designed for surgical procedure and operating room environment. Secondly, a participative team approach was adopted rather than a single external expert assessor, required from the traditional HEART, in line with previous studies in healthcare [5].

\subsection{Study Methodology}

This study took place at the Urology Department of one of the largest Italian hospitals. The starting point of the study was the development and validation of the BA-RARP task-analysis [15]. Once the task analysis was validated by the team of surgeons, the two most critical tasks were finally identified: "Isolation of lateral peduncles and of posterior prostate surface” (task 1), and “Anastomosis” (task 2). This decision came up from a preliminary focused literature review, further direct observational sessions, and experts' judgments elicitation (three surgeons were involved).

As previously mentioned, the modified HEART technique was applied with the aim of understanding what are the most relevant IFs and the degree of coverage with EPCs (Error Promoting Conditions) considered by the standard HEART technique. To achieve this aim, three surgeons, considered fully trained in the procedure, were asked to answer a specific questionnaire. In particular, referring separately to the first and second critical tasks, they had to identify which Influencing Factors exert a major influence on surgeon's performance. They were also asked to assess PoA and PoA* (percentage of PoA of the IFs attributable to the main corresponding EPC, estimated by the surgeons involved in this study) for each of the chosen IFs (see fig.1).

Afterwards, it was possible to identify the corresponding EPCs, calculate total PoA for each of them and the corresponding Assessed EPC Affect to proceed with HEART calculations about the Assessed Nominal Likelihood of Unreliability (ANLU). Subsequently, the \%CUs (contribution of unreliability) were calculated, in order to identify which are the IFs to be considered as the most critical ones, hence those requiring imperative remedial measures.

Finally, a Scenario Analysis was carried out to understand how ANLU may vary by mitigating the negative influence of different categories of Influencing Factors. In particular, personal, team, and organizational related factors were investigated by setting up three specific scenarios, for which new ANLU values were calculated by considering the impact of only the IFs relevant for the scenario.

\section{$3 \quad$ Results}

\subsection{Selection of generic task category}

The choice of the category could represent an issue for HEART application in healthcare, as underlined by previous studies [5] since some elements of the HEART 
task categories don't match the healthcare task under analysis. Since the focus of this analysis concerns the investigation of the most relevant IFs and the degree of coverage with HEART EPCs, we chose Category G for both tasks, i.e. "completely familiar, well-designed, highly practiced, routine task occurring several times per hour, performed to highest possible standards by highly-motivated, highly-trained \& experienced person, totally aware of implications of failure, with time to correct potential error, but without the benefit of significant job aids” [13]. Its Nominal Human Unreliability is 0.0004 .

\subsection{Correspondence Between EPCs and IFs}

The comparison between the two taxonomies was performed consulting the list of the EPCs proposed by Williams in his original document paper [13]. Some of them are not applicable to the surgical context, e.g. because they fit only with the nuclear context, and were excluded from the analysis (corresponding to EPCs code numbers 27, 28, 30, 31, 34, 38).

On the other hand, some of the IFs included in the new taxonomy describe recent phenomena, e.g. the use of digital devices and mobile phones in the operating room. Hence, only few of the factors resulted in a perfect matching, with a unique (one to one) clear link; for example "operator inexperience" and "stress" are conditions directly taken into account by both taxonomies. Many IFs of the surgical taxonomy are only partly captured by grouping more than one EPCs of the original Williams' taxonomy, so we assigned weights to each of them, by estimating to what extent a specific EPC describes the effect of each Influencing Factor. Table 1 shows a synthesis of the full spectrum of coverage and matching between Williams' taxonomy of EPCs and the proposed taxonomy of IFs in surgery.

According to the findings of the present study, the EPCs traditionally used in HEART method are not able to fully capture and explain the relevant organizational and personal IFs in a surgical context. This result confirms the importance of adapting HRA methods to the healthcare sector, and adds original detailed information on what are the most relevant factors that should be captured by an HRA method when applied to surgery in particular.

\subsection{Identification and assessment of IFs through HEART calculation}

To identify the influencing factors to be considered in HEART application we chose, as first selection criterion, the inclusion of those factors that were commonly identified by all the surgeons involved in the study and, as second criterion, those factors with no controversial assessment among surgeons. By averaging assessors PoA and PoA* values - for each IF - it was possible to balance surgeons' judgements, preventing overly optimistic or pessimistic results of the individual team member. The adoption of the average criterion is in accordance with previous HEART applications [5]. The selected Influencing Factors and their corresponding PoA and PoA* values, are shown in figure 1 for Task 1 and Task 2, whereas Figure 2 summarize the results of HEART application to the same tasks. 
Table 1. Matching and degree of coverage between the taxonomy of IFs in surgery and Williams’ EPCs.

Williams' EPCs

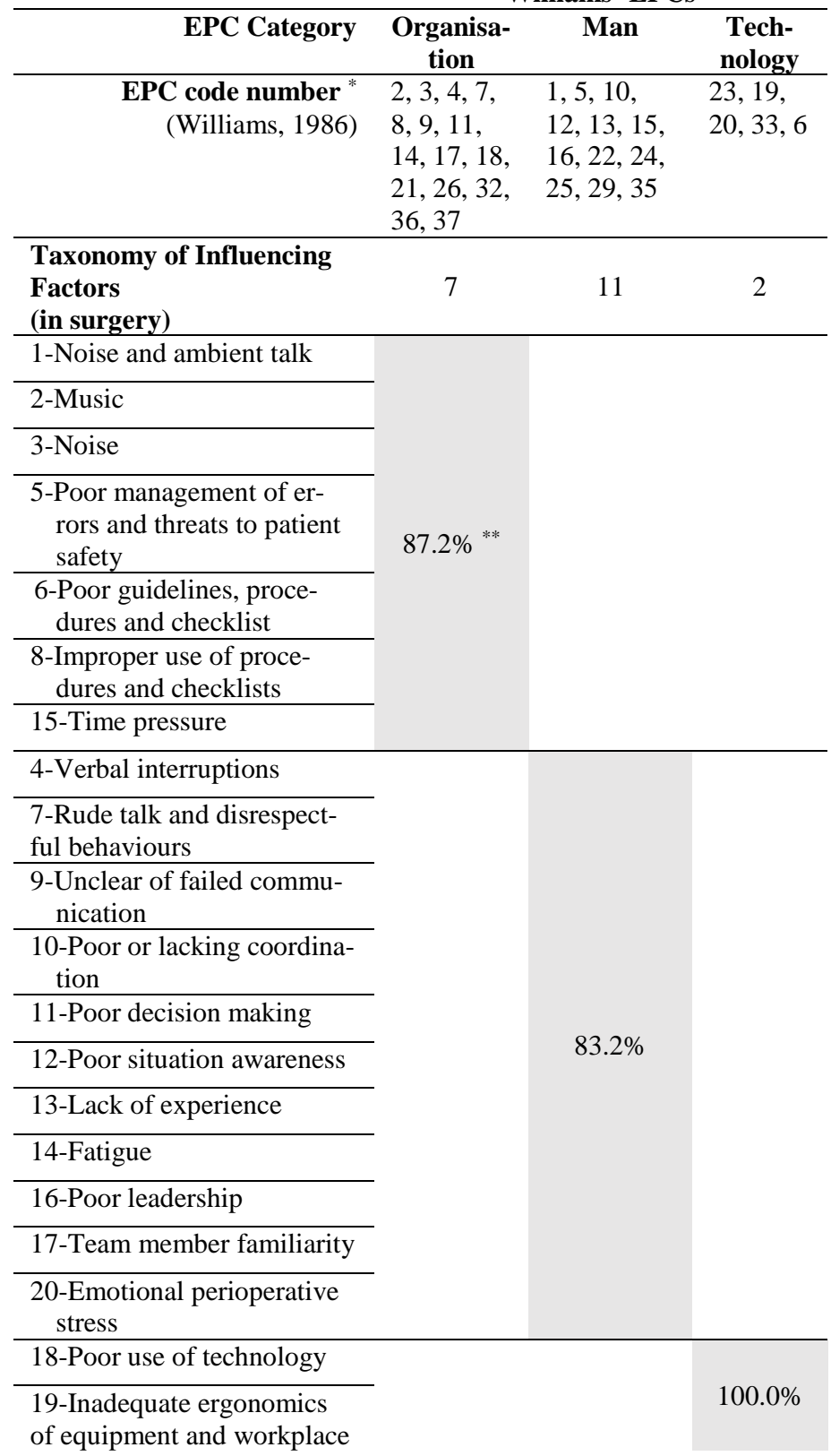

* EPCs n. 27, 28, 30, 31, 34, 38 are not applicable to the healthcare context.

** Organization-related EPCs (15) capture the $87.2 \%$ of the scope of IFs of the same type (7). 
As for Task 1, the three selected IFs were translated into seven EPCs showed in Figure 2. The most significant Influencing Factor is "Poor management of errors" (IF 5), which has a total \%CU of 0.49(see fig. 2). In particular, EPC 2 "Shortage of time available for error detection \& correction" is the corresponding EPC with the highest contribution to \%CU, having the greatest Multiplier (EPC Multiplier =11), and the largest value of Assessed EPC Affect. The second relevant IF is "Noise and Ambient Talk", which is linked to EPC 3 "Low signal to noise ratio". This EPC has the same value of PoA of the most significant one $(\mathrm{PoA}=58.67)$, but its Multiplier is slightly lower than the previous one. The last IF considered is "Poor coordination" (IF 10), with correspondence to EPC 10, which has the lowest Assessed EPC Affect and \%CU values (see fig. 2).

\begin{tabular}{|c|c|c|c|c|c|c|c|c|c|c|}
\hline \multicolumn{11}{|c|}{ TASK 1} \\
\hline IF & PoA1 & POA2 & POA3 & $\begin{array}{c}\text { PoA } \\
\text { average }\end{array}$ & EPC & & $P_{0} A^{* 1}$ & $\mathrm{PoA}^{* 2}$ & $\mathrm{PoA}^{*} 3$ & $\begin{array}{c}\mathrm{PoA}^{*} \\
\text { average }\end{array}$ \\
\hline 1 Noise\&ambient talk & 90 & 80 & 60 & 76.67 & 3 & Low signal to noise ratio & 60 & 56 & 60 & 58.67 \\
\hline 5 Poor management of errors & 70 & 70 & 70 & 70.00 & 2 & Shortage of time for errors detection & 50 & 56 & 70 & 58.67 \\
\hline 10 Poor coordination & 40 & 70 & 70 & 60.00 & 10 & Transfert knoledge from task to task & 10 & 63 & 70 & 47.67 \\
\hline \multicolumn{11}{|c|}{ TASK 2} \\
\hline IF & PoA1 & POA2 & POA3 & $\begin{array}{c}\begin{array}{c}\text { PoA } \\
\text { average }\end{array} \\
\text { a }\end{array}$ & EPC & & $\mathrm{POA}^{* 1}$ & $\mathrm{PoA}^{* 2}$ & $\mathrm{POA}^{*} 3$ & $\begin{array}{c}\mathrm{PoA}^{*} \\
\text { average }\end{array}$ \\
\hline $\begin{array}{ll}1 & \text { Noise\&ambient talk } \\
\end{array}$ & 80 & 70 & 60 & 70.00 & 3 & Low signal to noise ratio & 40 & 63 & 60 & 54.33 \\
\hline 5 Poor management of errors & 80 & 80 & 70 & 76.67 & 2 & Shortage of time for errors detection & 50 & 64 & 70 & 61.33 \\
\hline 9 Poor communication & 80 & 80 & 70 & 76.67 & 8 & Capacity overload & 10 & 48 & 70 & 42.66 \\
\hline 10 Poor coordination & 90 & 100 & 80 & 90.00 & 10 & Transfert knoledge from task to task & 20 & 50 & 80 & 50.00 \\
\hline
\end{tabular}

Figure 1. Final IFs selection for Task 1 and Task 2.

\begin{tabular}{|c|c|c|c|c|c|c|}
\hline \multicolumn{7}{|c|}{ TASK 1} \\
\hline IF & EPC & $\begin{array}{c}\text { EPC } \\
\text { Multiplier }\end{array}$ & PoA & $\begin{array}{l}\text { Assessed } \\
\text { EPC Affect }\end{array}$ & $\% \mathrm{Cu}$ & $\%$ CUIF \\
\hline \multirow{4}{*}{5 Poor management of errors } & 2 Shortage of time for errors detection & 11 & 58.67 & 6.867 & 0.33 & \multirow{4}{*}{0.49} \\
\hline & 7 No means reversing unintended actions & 8 & 2.83 & 1.198 & 0.058 & \\
\hline & 12 Mismatch: perceived \& real risks & 4 & 5.665 & 1.170 & 0.056 & \\
\hline & 18 Conflict: long \&immediate objectives & 2.5 & 28.325 & 1.042 & 0.05 & \\
\hline 1 Noise \& Ambient talk & 3 Low signal to noise ratio & 10 & 58.67 & 6.280 & 0.302 & 0.3 \\
\hline \multirow{2}{*}{10 Poor coordination } & 10 Transfer know ledge from task to task & 5.5 & 47.67 & 3.145 & 0.151 & \multirow{2}{*}{0.2} \\
\hline & 25 Unclear allocation of responsibilities & 1.6 & 12.33 & 1.074 & 0.052 & \\
\hline \multicolumn{7}{|c|}{ TASK 2} \\
\hline IF & EPC & $\begin{array}{c}\text { EPC } \\
\text { Multiplier }\end{array}$ & PoA & $\begin{array}{l}\text { Assessed } \\
\text { EPC Affect }\end{array}$ & $\% \mathrm{Cu}$ & $\%$ CUIF \\
\hline \multirow{4}{*}{5 Poor management of errors } & 2 Shortage of time for errors detection & 11 & 61.33 & 7.133 & 0.259 & \multirow{4}{*}{0.39} \\
\hline & 7 No means reversing unintended actions & 8 & 3.84 & 1.268 & 0.046 & \\
\hline & 12 Mismatch: perceived \& real risks & 4 & 7.67 & 1.230 & 0.045 & \\
\hline & 18 Conflict: long \&immediate objectives & 2.5 & 3.84 & 1.058 & 0.038 & \\
\hline \multirow{2}{*}{9 Poor communication } & 5 No means of conveying info & 8 & 34 & 3.380 & 0.123 & \multirow{2}{*}{0.24} \\
\hline & 8 Capacity overload & 6 & 42.67 & 3.133 & 0.114 & \\
\hline 1 Noise \& Ambient talk & 3 Low signal to noise ratio & 10 & 54.33 & 5.890 & 0.214 & 0.21 \\
\hline \multirow{2}{*}{10 Poor coordination } & 10 Transfer know ledge from task to task & 5.5 & 50 & 3.250 & 0.118 & \multirow{2}{*}{0.16} \\
\hline & 25 Unclear allocation of responsibilities & 1.6 & 40 & 1.240 & 0.045 & \\
\hline
\end{tabular}

Figure 2. Results of HEART application to the task "Isolation of lateral peduncles and of posterior prostate surface” (Task 1 and Task 2).

The four IFs selected for Task 2 were translated into nine EPCs, as showed in Figure 2. Also in this case, IF 5 "Poor management of errors", primarily referred to EPC 2 ("Shortage of time available for error detection \& correction") resulted to be the most 
critical one, having the greatest value of Assessed EPC Affect and Percentage Contribution to Unreliability (\%CU = 0.39)(see fig. 2). The second IF is "Unclear communication", which is an IF that was considered as not relevant for Task 1 by the surgical team. This IF is partly captured by EPC 5 and EPC 8 (see fig. 2); they both have medium EPC Multiplier and PoA values and contribute to a \%CU value of 0.24 (see fig. 2). Third and fourth positions are covered by IF $1(\% \mathrm{CU}=0.21)$ and IF $10(\% \mathrm{CU}=$ 0.16 ), respectively (see fig. 2).

Starting from the same task category (G), the final ANLU (Assessed Nominal Likelihood of Unreliability) value for task 2 resulted to be greater than task 1, since the former is subject to a larger number of IFs. Furthermore, the two tasks under analysis share the same most critical IF, i.e. "Shortage of time available for error detection \& correction” (EPC 2_IF 5), as demonstrated by the corresponding EPC Percentage Contribution to Unreliability of $0.33 \%$ for Task 1 and $0.26 \%$ for Task 2 .

\subsection{Sensitivity Analysis on single IFs and reference scenarios}

A Scenario Analysis was finally performed to investigate the sensitivity of critical tasks to different conditions. To this end, the maximum variations of the ANLU was estimated under different combinations of IFs and finally compared to the Nominal Human Unreliability for the chosen Generic Task (NHU_G $=0.0004)$.

A first level analysis dealt with the sensitivity of ANLU to the IFs deemed as the most relevant according to surgeons' perception. ANLU values for each IF was estimated for both Task 1 and Task 2, using surgeons' averaged assessment of PoA values (Figure 2). The greatest range of ANLU variation results from "Poor management of errors” (IF 5) for both Task 1 and Task 2 (Table 2).

Table 2. Range of variation of ANLU due to the effect of relevant IFs

\begin{tabular}{llclc}
\hline & $\begin{array}{c}\text { Noise and } \\
\text { Ambient } \\
\text { Talk } \\
\text { (IF 1) }\end{array}$ & $\begin{array}{c}\text { Poor } \\
\text { manage- } \\
\text { ment of } \\
\text { errors } \\
\text { (IF 5) }\end{array}$ & $\begin{array}{c}\text { Unclear } \\
\text { Communica- } \\
\text { tion } \\
\text { (IF 9) }\end{array}$ & $\begin{array}{c}\text { Poor Co- } \\
\text { ordina- } \\
\text { tion } \\
\text { (IF 10) }\end{array}$ \\
\hline Range of & $0.00040-$ & $0.00040-$ & Not & $0.00040-$ \\
$\begin{array}{l}\text { ANLU for } \\
\text { Task 1 }\end{array}$ & 0.00250 & 0.00400 & relevant & 0.00135 \\
\hline $\begin{array}{l}\text { Range of } \\
\text { ANLU for } \\
\text { Task 2 }\end{array}$ & $0.00040-$ & $0.00040-$ & $0.00040-$ & $0.00040-$ \\
\hline
\end{tabular}

A second level of analysis was performed with reference to three different scenarios. A scenario corresponds to the selection of a meaningful combination of relevant IFs, considering three main categories of factors: personal, team and organizational related (Table 3). The Reference Scenarios represent a way of modelling with HEART plausible situations in the operating room. In the following, the three scenarios are briefly described by making use of the information obtained from direct observations and interviews with surgeons. 
The first scenario considered is related to personal factors that occur during the execution of a surgical procedure. "Unclear Communication" (IF 9) and "Poor Coordination" (IF 10) were identified by the surgeons as the IFs having a strong impact on surgeon's performance, and widely detected during the observational phase of the study. According to surgeons' judgements, “Unclear Communication” (IF 9) was selected for Task 2 only. The new value of ANLU for Task 1 is 0.00135 , which corresponds to $98.4 \%$ reduction from the starting condition of the case study (ANLU = $0.0851)$ and increase of two order of magnitude from the NHU (0.0004). The new value of ANLU for Task 2 is 0.0171 , corresponding to $98.3 \%$ reduction from the reference case and increase of three orders of magnitude from the NHU (0.0004). Figure 3 shows the Human Unreliability Rate when poor personal factors influence the surgeon's performance; it also highlights that the reliability of Task 2 is more sensitive to poor personal conditions than Task 1.

Table 3. Characteristics of the three Reference Scenarios as combination of relevant IFs taken into consideration.

\begin{tabular}{clccc} 
& \multicolumn{3}{c}{ Reference Scenarios } \\
\cline { 3 - 5 } & $\begin{array}{c}\text { Poor } \\
\text { personal } \\
\text { conditions }\end{array}$ & $\begin{array}{c}\text { Poor } \\
\text { team con- } \\
\text { ditions }\end{array}$ & $\begin{array}{c}\text { Poor } \\
\text { Organizational } \\
\text { conditions }\end{array}$ \\
\hline 1 & $\begin{array}{l}\text { Noise and Ambient } \\
\text { Talk }\end{array}$ & $\mathrm{X}$ & \\
\hline 5 & $\begin{array}{l}\text { Poor management } \\
\text { of errors }\end{array}$ & $\mathrm{X}$ & $\mathrm{X}$ & $\mathrm{X}$ \\
\hline 9 & $\begin{array}{l}\text { Unclear } \\
\text { Communication }\end{array}$ & $\mathrm{X}$ & $\mathrm{X}$ & \\
\hline 10 & Poor Coordination & $\mathrm{X}$ & & \\
\hline
\end{tabular}

The second scenario is the one concerning the influence of team factors on surgeon's performance. "Noise and Ambient Talk" (IF 1), "Unclear Communication” (IF 9), and "Poor Coordination" (IF 10) were included in this second scenario. Team members are key elements of the system. Poor coordination and unclear communication can either occur explicitly or implicitly. Team members can intentionally communicate or they can anticipate, assist and adjust without verbal instructions, relying on a shared understanding of the task and of the situation. Team members are continuously involved in reciprocal process to send/receive information that shapes and re-shapes team's attitudes, behaviors, and situational awareness. The new value of ANLU for Task 1 is 0.00848 , which corresponds to $90 \%$ reduction from the starting condition of the case study and the $2021 \%$ increase from NHU (0.0004). The new value of ANLU for Task 2 is 0.1005 , which corresponds to $89.9 \%$ reduction from the reference case and four orders of magnitude increase from the NHU (0.0004).

According to surgeons' perception of the most relevant IFs for the considered surgical procedure and tasks, the third scenario is described by "Poor management of errors" (IF 5) only. It refers to the scenario in which procedures to share information promptly and openly about errors are compromised. The new ANLU value for Task 1 is 0.00401 , which corresponds to $95.3 \%$ reduction from the starting condition of the case study (ANLU $=0.0851$ ) and the increase of one order of magnitude from 
the NHU (0.0004). The new ANLU value for Task 2 equals to 0.00471, which corresponds to $99.5 \%$ reduction of ANLU and 1077\% increase from the NHU (0.0004). The graph reported in Figure 3 shows that the case of poor organizational conditions is the scenario with the lowest impact on surgeons' performance (measured by HEART as Human Unreliability rate), whereas the case of poor team conditions represents the worst scenario for both tasks.

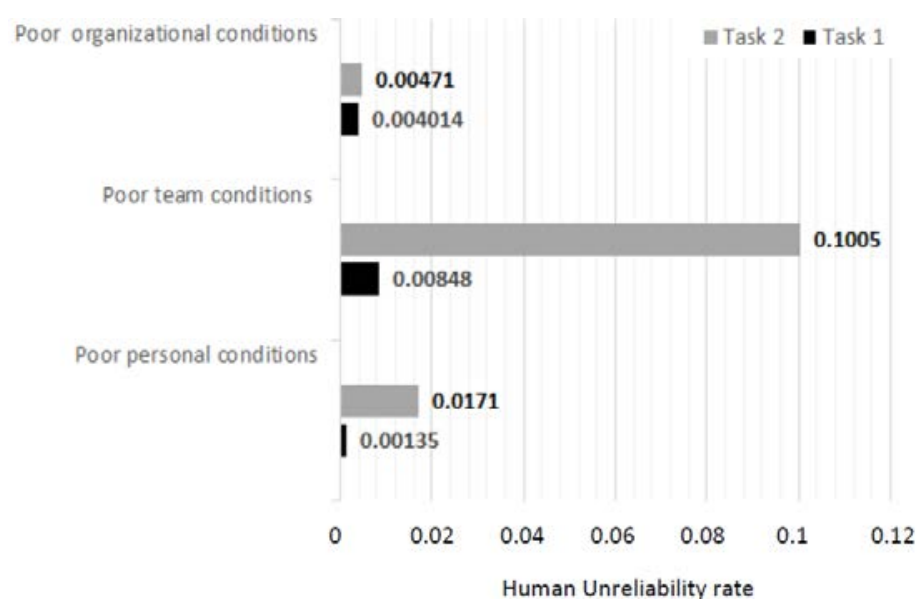

Figure 3. Scenario Analysis.

\section{Conclusions}

This study aimed at contributing to the development of HRA methodologies in healthcare, and surgery in particular, as a quantitative approach to the identification of risk factors and appropriate mitigation measure against surgical adverse events.

Furthermore, although the study confirms the value of adopting HEART technique in the context of Clinical Risk Management, results support the argument in favor of a deep adaptation and translation of HRA techniques to the healthcare context. In this regard, the present study offers a systematic approach to matching and quantification of the correspondence between a taxonomy of IFs in surgery [17] and the Williams' taxonomy of EPCs normally used in HEART applications [13]. The assessment of surgeon's reliability/unreliability in robotic surgery was assumed as a significant case study, since it is an increasing innovative mini-invasive surgical approach, whose advanced technology needs to be managed in order to limit the possible new error pathways due to the complexity of human-machine interactions. The major role of team related factors in determining surgical performance, when compared to other personal or organizational factors, is another original contribution of the present study. Further developments of this research may concern: i) the investigation of an entire surgical procedure, so as to also consider task dependencies and error recovery procedures; ii) the adoption of HRA techniques in the context of surgeons training and proficiency assessment. 


\section{References}

[1] Cagliano, A.C., Grimaldi, S. and Rafele, C. (2011), “A systemic methodology for risk management in healthcare sector”, Safety Science, Elsevier Ltd, Vol. 49 No. 5, pp. 695-708.

[2] Lyons, M. (2009), "Towards a framework to select techniques for error prediction: supporting novice users in the healthcare sector.”, Applied ergonomics, Elsevier Ltd, Vol. 40 No. 3, pp. 379-95.

[3] Lyons M., Adams, S., Woloshynowych, M., Vincent, C. (2004), "Human reliability analysis in healthcare: A review of techniques", International Journal of Risk \& Safety in Medicine, Vol. 16 No. 4, pp. 223-237.

[4] Verbano, C. and Turra, F. (2010), “A human factors and reliability approach to clinical risk management: Evidence from Italian cases”, Safety Science, Vol. 48 No. 5, pp. 625-639.

[5] Chadwick, L. and Fallon, E.F. (2012), "Human reliability assessment of a critical nursing task in a radiotherapy treatment process.”, Applied ergonomics, Vol. 43 No. 1, pp. 89-97.

[6] Cuschieri, A. (2000), "Human reliability assessment in surgery--a new approach for improving surgical performance and clinical outcome.”, Annals of the Royal College of surgeons of England, pp. 83-87.

[7] Cuschieri, A. and Tang, B. (2010), "Human reliability analysis (HRA) techniques and observational clinical HRA.", Minimally invasive therapy \& allied technologies : MITAT : official journal of the Society for Minimally Invasive Therapy, Vol. 19 No. 1, pp. 12-7.

[8] Malik, R., White, P.S. and Macewen, C.J. (2003), "Using human reliability analysis to detect surgical error in endoscopic DCR surgery”, Clinical Otolaryngology and Allied Sciences, Vol. 28 No. 5, pp. 456-460.

[9] Miskovic, D., Ni, M., Wyles, S.M., Kennedy, R.H., Francis, N.K., Parvaiz, A., Cunningham, C., et al. (2013), "Is Competency Assessment at the Specialist Level Achievable? A Study for the National Training Programme in Laparoscopic Colorectal Surgery in England.”, Annals of surgery, Vol. 257 No. 3, pp. 476-482.

[10] Boring, R.L. (2010), "How Many Performance Shaping Factors are Necessary for Human Reliability Analysis?"

[11] Fallon, E.F., Chadwick, L. and Putten, W.J. Van Der. (2015), “An Analysis of the Impact of Trends in Automation on Roles in Radiotherapy Using Function Allocation”, Human Factors, Vol. 3 No. Ahfe, pp. 234-241.

[12] Ward, J., Teng, Y., Horberry, T. and Clarkson, P.J. (2004), "HEALTHCARE HUMAN RELIABILITY ANALYSIS - BY HEART”, Vol. 38, pp. 287-288.

[13] Williams, J.C., 1986. HEART - a proposed method for assessing and reducing hu-man error. In: 9th Advances in Reliability Technology Symposium. University of Bradford.

[14] Al Naami, M., Anjum, M. N., Aldohayan, A., Al-Khayal, K., \& Alkharji, H. (2013). Robotic general surgery experience: a gradual progress from simple to more complex procedures. The International Journal of Medical Robotics and Computer Assisted Surgery, 9(4), 486-491.

[15] Galfano, A., Ascione, A., Grimaldi, S., Petralia, G., Strada, E. and Bocciardi, A.M. (2010), "A new anatomic approach for robot-assisted laparoscopic prostatectomy: A feasibility study for completely intrafascial surgery”, European Urology, European Association of Urology, Vol. 58 No. 3, pp. 457-461.

[16] Galfano, A., Di Trapani, D., Sozzi, F., Strada, E., Petralia, G., Bramerio, M., Ascione, A., et al. (2013), "Beyond the learning curve of the Retzius-sparing approach for robot-assisted laparoscopic radical prostatectomy: Oncologic and functional results of the first 200 patients with $\geq 1$ year of follow-up”, European Urology, European Association of Urology, Vol. 64 No. 6, pp. 974-980.

[17] Onofrio, R., Trucco, P. and Torchio, A. (2015), “Towards a Taxonomy of Influencing Factors for Human Reliability Analysis (HRA) Applications in Surgery”, Procedia Manufacturing, Vol. 3, pp. 144-151. 